\title{
“IMPLEMENTACIÓN DE ESTRATEGIAS PARA LA ENSEÑANZA DE CIENCIAS NATURALES UTILIZANDO RECURSOS TECNOLÓGICOS"
}

\author{
Presentación: Comunicación Oral \\ Giovanna Silva Santis ${ }^{1}$; Lastenia Ugalde $\mathrm{Meza}^{2}$
}

\section{Resumen}

Este trabajo de investigación se compone de dos etapas fundamentales: La primera consistió en la elaboración de los recursos didácticos informatizados con temáticas relacionadas con el análisis y caracterización del suelo; temáticas que forman parte del curriculum de $6^{\circ}$ año de enseñanza básica. El desarrollo del material apunta a la incorporación de conceptos básicos de ciencias naturales, además de introducir a los estudiantes en los conceptos medioambientales. La segunda etapa la implementación consistió en propuestas para la transformación de suelos a través de la fabricación de una compostera.

Palabras Clave: compostera, suelos, tic, enseñanza básica

\section{Introducción}

La sociedad desde hace ya varios años muestra preocupación por las consecuencias tanto presentes como futuras del daño causado al medio ambiente por obra del mismo ser humano.

Los esfuerzos por crear conciencia de la importancia de tener una cultura de cuidado medioambiental parecen no ser suficientes y, es ahí cuando la comunidad educativa debe hacerse parte y convertirse en el factor de cambio, que eduque futuros ciudadanos proactivos en este menester.

Los cambios en las sociedades deben hacerse desde sus bases; es decir, desde los estudiantes de enseñanza básica. Es sabido que los alumnos pequeños aprenden desde las primeras etapas por modelaje, es decir, por imitación; y el hecho que pasen la mayor parte del día en los establecimientos escolares hacen imprescindible que tanto, profesores como la comunidad escolar e su totalidad enseñen mediante el ejemplo conductas de cuidado del medioambiente.

\footnotetext{
1 Pedagogía en Química y Ciencias, Univerisidad de Playa Ancha de las Ciencias de la Educación, giovanna.silva.santis@gmail.com

${ }^{2}$ Doctora en Química, Universidad Católica de Chile, lastenia.ugalde@upla.cl
} 


\section{Objetivo General:}

Motivar a los alumnos al estudio de las ciencias a través del análisis de suelos mediante el uso de TICs y actividades experimentales.

\section{Objetivos Específicos}

Elaborar material didáctico informatizado (videos), para aportar al aprendizaje de conceptos básicos del suelo, además de crear protocolos de análisis de suelo.

$>$ Evaluar la percepción del uso de las Tic de los estudiantes de enseñanza básica.

$>$ Preparación y aplicación de materiales de uso cotidiano para el análisis físico de suelo

$>$ Incorporar en los estudiantes el concepto de Compost como una intervención positiva para modificar el suelo.

\section{Fundamentación Teórica}

Dentro de este trabajo se busca validar el uso de vídeos como estrategia de motivación para los estudiantes hacia un aprendizaje más significativo y que perdure en el tiempo. Si tomamos a la motivación como el estímulo emocional que nos hace actuar y que puede una necesidad o un impulso que activa ciertas conductas entonces, al saber lo que motiva a nuestros estudiantes habrá mayores probabilidades de que se alcancen las metas (Dalton, Hoyle, \& Watts, 2007).

Sin embargo, para poder motivar a nuestros estudiantes hace falta saber cuáles son sus intereses actuales, para poder ligarlos con los contenidos propios de nuestras asignaturas. Es allí donde intentamos ligar lo tecnológico, tan en boga actualmente, pues las nuevas generaciones manejan las tecnologías de manera muy natural, ya que se han criado con ellas el estudio de suelos en este caso.

En ese ámbito la educación se encuentra en deuda, pues no se ha hecho cargo de aquello, ya que se sigue enseñando como hace 20 o 30 años atrás cuando los estudiantes no son os mismos que en aquella época.

Además del uso de vídeos, que por sí solo no parece suficiente para la motivación de los jóvenes mas solo logra causar mayor interés, es que se quiso dar un sentido a lo estudiado al ligarlo al mejora de suelos para hacerlos aptos para el cultivo a través del uso de compost y la fabricación de este compost a través de la selección de los desechos. Entonces, recién allí cuando logramos a 
través de los vídeos captar la atención de los estudiantes y al darle luego un sentido a lo aprendido es que logramos motivar realmente a los estudiantes.

Cuando se analiza el área de la Química, las TIC han dado valiosos aportes como herramienta de trabajo para la enseñanza y el aprendizaje de esta disciplina; entre los aportes según (Cabero J., 2007) están la posibilidad de realizar simulaciones de procesos y prácticas de laboratorio, el ayudar a la modelización y representación gráfica de determinados fenómenos, el apoyo a la activación y desactivación de moléculas en tres dimensiones, realizar relaciones visuales entre los modelos moleculares en dos o tres dimensiones e intercambio de información

Nadie discute que las capacidades intelectuales influyen en el mayor o menor nivel de aprendizaje de un alumno y, sobre todo, en la facilidad de aprendizaje. Pero para que estas capacidades se pongan en juego es preciso que previamente el estudiante haya decidido hacerlo. Demasiados alumnos de secundaria con capacidades suficientes para conseguir los objetivos de aprendizaje correspondientes a su nivel no llegan a lograrlo porque no tienen interés (García, 2005)

Entonces lo que se pretende finalmente con esta intervención es poder lograr mediante la motivación mejorar los resultados de los estudiantes al poder desarrollar sus capacidades al momento de aprender. Esta motivación constará de una etapa inicial en la que se captará la atención de los estudiantes mediante la utilización de vídeos informatizados, luego de una contextualización, en este caso medioambiental de los alcances de lo estudiado y finalmente de cómo pueden haciendo uso de lo aprendido hacer la diferencia en su comunidad.

\section{Metodología}

La metodología propuesta para la primera etapa es el uso de videos, como recursos didácticos informatizados que ayuden a los alumnos a incorporar conceptos básicos del suelo, y también que puedan ver protocolos de análisis de suelo. Dichos análisis han sido montados con materiales de uso cotidiano, introduciendo además el concepto de reciclaje. Todos estos protocolos de análisis del suelo permiten llevar a los alumnos hacia una definición y clasificación de los mismos y, por lo tanto, los alumnos podrán discriminar y diferenciar entre un suelo apto para el cultivo de uno empobrecido, y con ello estimar cuales podrían ser los mecanismo de intervención para mejorar dichos suelos. 


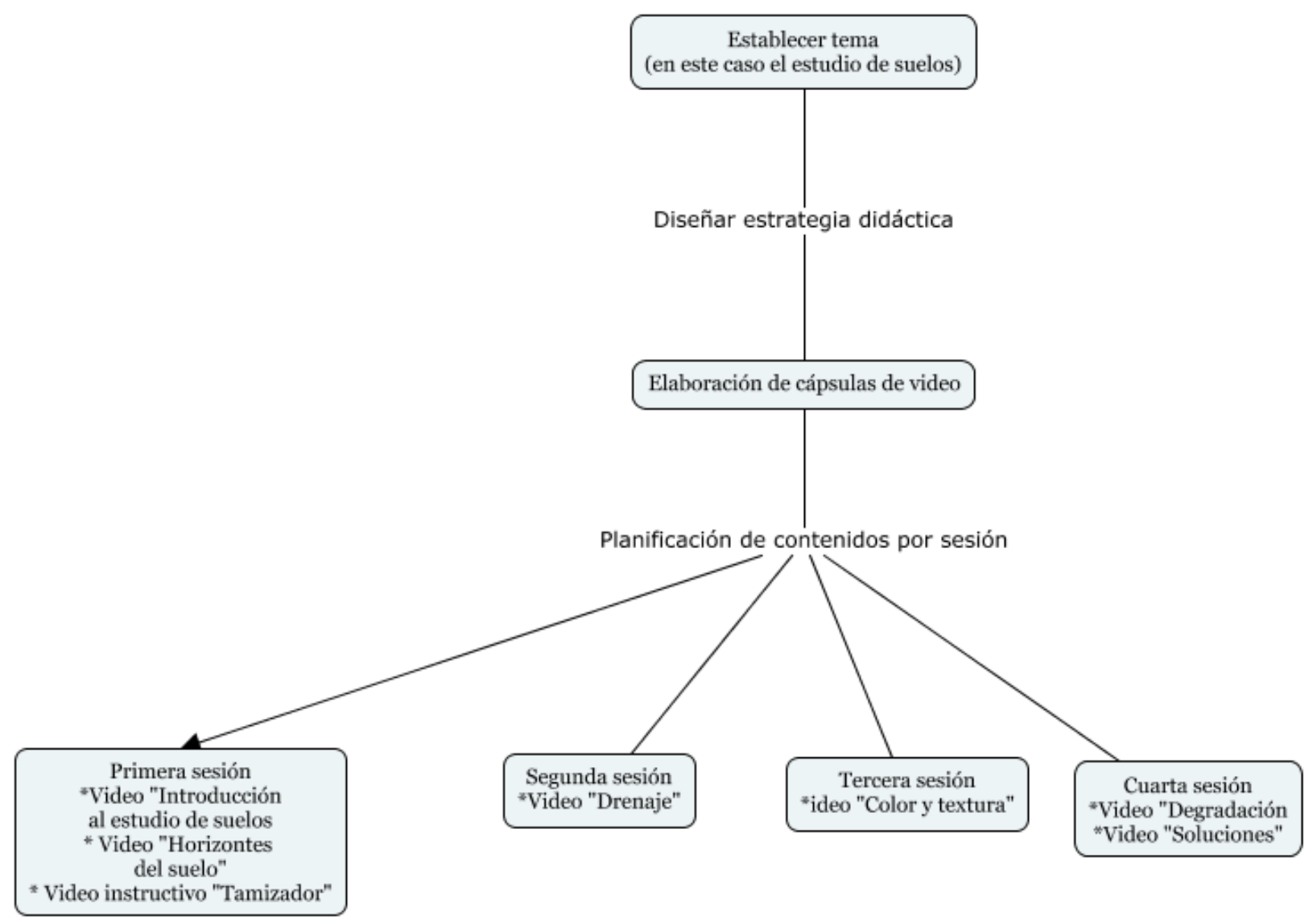

La segunda etapa, de mejora de suelos, se enseñará de manera didáctica (a través de los videos) la forma de hacer y utilizar compost para el tratamiento de suelos en busca de logran un aumento en su fertilización, ya que, hemos podido verificar que de esta manera se tiene una mejor llegada a los estudiantes, logrando un mayor grado de motivación, mejorando los niveles de trabajo colaborativo y aliviando la carga de trabajo de los profesores (Castaño \& Cabero, 2014), dado que los videos pueden ser reproducidos por los estudiantes las veces que sean necesarias.

Este estudio se realizó en la Escuela estatal América de Playa Ancha, Valparaíso, específicamente en el curso de $6^{\circ}$ año básico "A", que cuenta con un número de estudiantes aproximado de 20-25 alumnos. La intervención se realizó en las horas correspondientes a la asignatura de ciencias naturales, esto correspondió a 4 sesiones de 90 minutos cada una.

Elaboración de recursos didácticos informatizados:

Los vídeos fueron realizados en el programa Powtoon ${ }^{\circledR}$, donde se elaboraron cápsulas de vídeo de no más de 3 minutos para poder mantener la atención de los estudiantes de principio a fin 
y no abrumarlos con exceso de información.

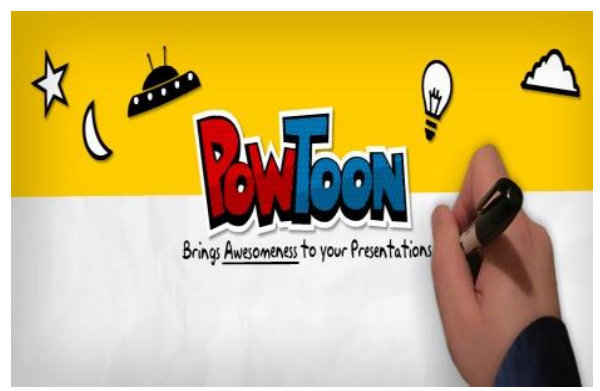

Figura1. Programa Powtoon®, elaboración de cápsulas de vídeo

Además para complementar los vídeos se realizaron audios que luego fueron agregados a los vídeos haciendo uso del programa Audacity®, para lograr resultados óptimos para la motivación de los estudiantes pues tenían que enfocar su atención visual y auditiva en lo que se estaba presentando.

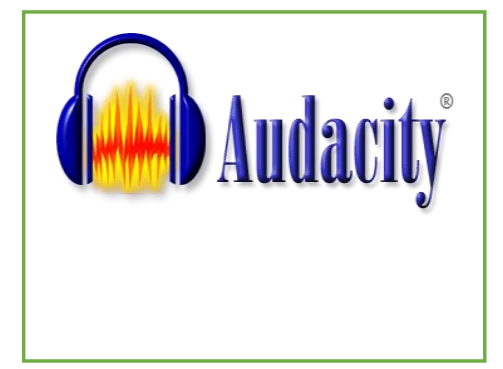

Figura 2. Programa Audacity®, elaboración de audios.

En la primera clase se expuso el vídeo “introducción estudio suelos” que resumía las características que serían estudiadas durante las sesiones de la intervención. Después, durante la misma clase se exhibió el vídeo "horizontes del suelo" que explicaba cada uno de los horizontes que componen el suelo y sus características, para terminar con el vídeo "tamizador” que explicaba la elaboración paso a paso de un tamizador con materiales caseros para la caracterización de los suelo desde el tamaño de sus partículas. 


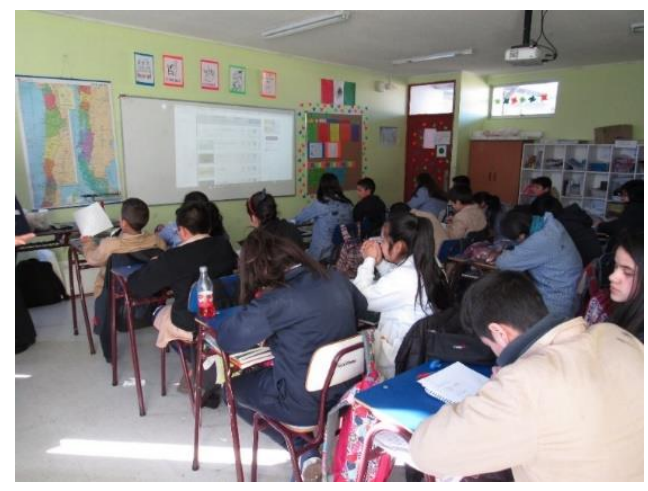

Figura 3. Desarrollo intervención, primera sesión

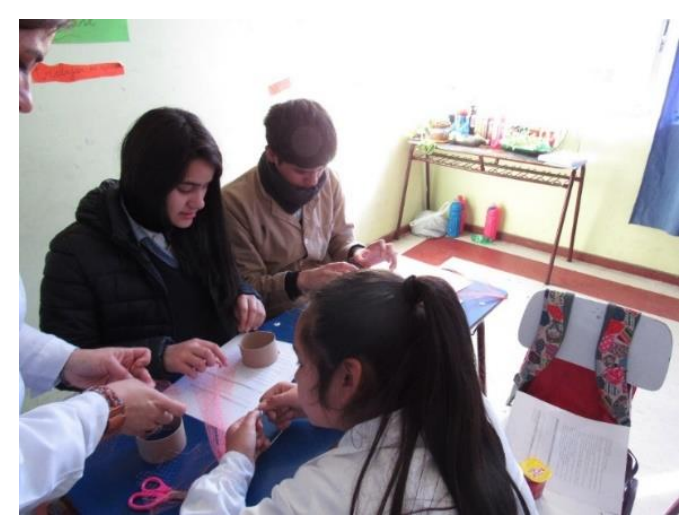

Figura 4. Alumnos trabajando en la elaboración de un tamizador luego de ver el vídeo introductorio.

La segunda clase se exhibió la cápsula de vídeo "drenaje" en la cual se explicaba el paso a paso de la elaboración de un dispositivo para la medición de la velocidad de drenaje de los distintos suelos. La clase siguiente se hizo uso de la cápsula de vídeo "color y textura" en la que se explicaban las características según los distintos colores de los suelos y la textura que podían poseer y, además se daban instrucciones para la elaboración de un muestrario donde podían compara dos tipos de suelo distintos.

Finalmente en la última clase se expusieron los vídeos "degradación” y "soluciones", que planteaban el daño que se puede causar a los suelos y las formas en que este daño se puede revertir a través de la separación de desperdicios y elaboración de compost respectivamente. Lo que va en cumplimiento con lo planteado en el inicio de la investigación con respecto a la segunda etapa en la cual se contextualiza lo aprendido y se da un sentido, para que el alumno comprenda el por qué se enseña lo anterior.

Cabe mencionar que en cada sesión, luego de la exhibición de los vídeos se realizaba un cuestionario de no más de 5 preguntas para medir si las cápsulas estaban siendo de utilidad para el estudio de la unidad. 
1. Describa los suelos que analizó según color y textura

2. El color oscuro en la tierra, demuestra la presencia de...

3. ¿Cuál es la relación entre color y textura en los suelos?

4. ¿De qué depende el color de la arcilla?
a) De la cantidad de materia orgánica
b) Del tipo de mineral que esté presente
c) Del día del año

Figura 5. Muestra de cuestionario utilizado

\section{Resultados y discusión}

Los resultados después de la aplicación del primer vídeo se enfocaron en evaluar la percepción del uso del recurso didáctico (video), con lo cual se obtuvo el grado de aceptación de los estudiantes respecto de utilización de las cápsulas de vídeo para la introducción de contenidos y descripción de actividades. El análisis mostró resultados positivos de la percepción del recurso didáctico utilizado (Fig,6)

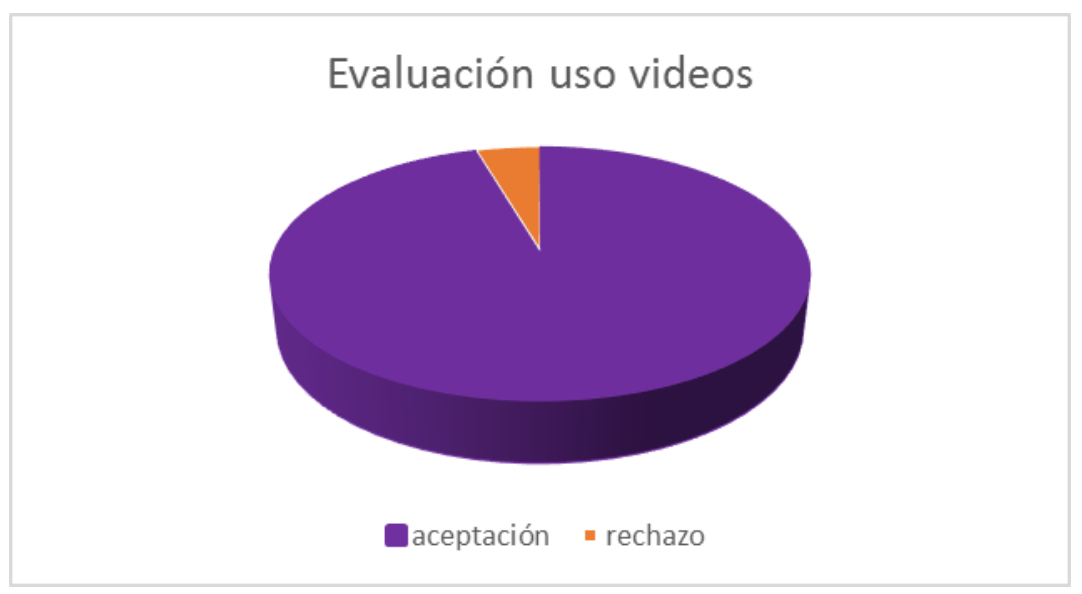

Figura 6. Gráfico evaluación de percepción al recurso didáctico

Cabe mencionar que se hicieron evaluaciones de medición de comprensión de contenidos, 
donde destaca la evaluación final, que midió si los estudiantes lograban diferenciar de una serie de materiales de desecho cuáles de ellos correspondían a desechos orgánicos y cuales podían ser utilizados como material de reciclaje. Los resultados se muestran la tabla siguientes:

Tabla 1: Respuestas cuestionario final, donde la "alternativa a" corresponde a material de reciclaje y la "alternativa b" a material orgánico"

\begin{tabular}{|l|r|r|r|}
\hline Material & alternativa a & alternativa $\mathbf{b}$ & $\mathbf{n} / \mathbf{r}$ \\
\hline cáscara de huevo & 2 & 13 & 0 \\
\hline botellas plásticas & 14 & 1 & 0 \\
\hline papel de diario & 13 & 2 & 0 \\
\hline latas & 14 & 1 & 0 \\
\hline papas en descomposición & 2 & 13 & 0 \\
\hline vidrios rotos & 13 & 2 & 0 \\
\hline flores marchitas & 1 & 14 & 0 \\
\hline cartones & 14 & 0 & 1 \\
\hline
\end{tabular}

En el siguiente gráfico (Fig.8) muestra el grado de aprendizaje de los contenidos llevados a la vida cotidiana, donde los estudiantes fueron capaces de discriminar de un set de distintos materiales (mencionado en la tabla 1) cuales correspondíann a materiales de desecho y cuáles puedían ser utilizados para la elaboración de compost para la mejora de suelos. Alcanzando el 90\% las respuestas correctas y solo el $10 \%$ errando.

\section{Resultado final intervención}

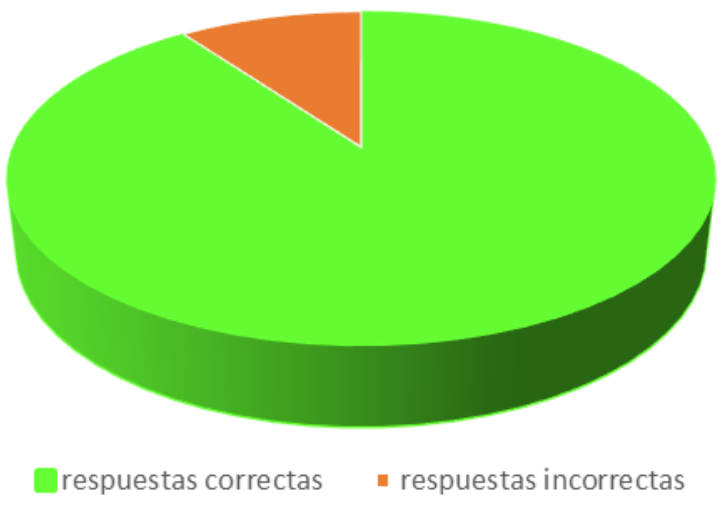

Figura 8. Gráfico respuestas cuestionario final 


\section{Conclusiones}

En primer lugar podemos decir que se logró a través de los programas anteriormente mencionados ("Powtoon" y "Audacity") la elaboración de material didáctico informatizado, el que fue utilizado en todas las sesiones según el mapa conceptual presentado en el punto de metodología.

La evaluación realizada por los alumnos de enseñanza básica que fueron partícipes de la intervención fue muy positiva, llegando al 95\% de aceptación de la utilización del material, en este caso cápsulas de video.

Se prepararon todas las actividades experimentales con materiales de uso cotidiano. Estas actividades fueron llevadas a cabo gracias a los videos inductores donde se explicaba el paso a paso de lo que se debía hacer para agilizar el proceso y optimizar los tiempos de clases.

Al finalizar el proceso, los alumnos lograron incorporar el concepto de compost, dado que en las actividades desarrolladas para tal fin fueron realizadas con éxito por los alumnos.

Al término de la intervención, los alumnos se mostraron muy motivados por seguir esta dinámica de enseñanza, pues les pareció interesante y les permitía participar de manera más activa tanto de las actividades como del proceso de aprendizaje.

\section{Referencias}

- Castaño, C., \& Cabero, J. (2014). Enseñar y aprender en entornos m-learning. Madrid: Editorial Síntesis S.A.

- Dalton, M., Hoyle, D. G., \& Watts, M. W. (2007). Relaciones Humanas. México: International Thomson Editores, S.A.

- García, F. (2005). Motivar para el aprendizaje desde la actividad orientadora. Madrid: SECRETARIA GENERAL TÉCNICA Subdivisión General de Información y Publicaciones.

- Cabero, J. (2007). Las Tics en la enseñanza de la Química: aportaciones desde la Tecnología Educativa, en BODALO, A. y otros (eds.) (2007): Química: vida y progreso, Murcia, Asociación de químicos de Murcia, Universidad de Sevilla. 